\title{
A Computer-Aided System for the Detection of Prostate Cancer Based on Magnetic Resonance Image Analysis
}

\author{
D. Ampeliotis*, A. Antonakoudi ${ }^{\dagger}$, K. Berberidis*, E. Z. Psarakis* and A. Kounoudes ${ }^{\dagger}$ \\ * Computer Engineering and Informatics Department, University of Patras, 26500, Rio-Patras, Greece \\ $\dagger$ Computing and Information Systems Department, The Philips College, 28008, Nicosia, Cyprus
}

\begin{abstract}
This paper presents an overview of a computeraided system for the detection of carcinomas in the prostate gland. The proposed system incorporates information from two different types of Magnetic Resonance Images (MRIs), namely the T2-weighted morphological images and the T1-weighted Dynamic Contrast Enhanced (DCE) images, to extract discriminative features that will be used in the training phase of a classification algorithm for the differentiation between malignant and benign tissue. The resulting feature vectors are also used for the assessment of new patient cases. The pattern recognition scheme is based on Probabilistic Neural Networks (PNNs). The parameters of the PNNs are estimated using the ExpectationMaximization (EM) algorithm. The performance of the proposed computer-aided detection system is evaluated through training and testing on several patient cases, whose condition has been previously assessed through ultrasound-guided biopsy and MRI examination.
\end{abstract}

\section{INTRODUCTION}

Prostate cancer is a commonly diagnosed malignancy in men in developed countries [1]. The detection of prostate carcinomas at an early stage results in better treatment, increasing the patient survival rate. Well known tests such as the prostate specific antigen test (PSA), and digital rectal examination (DRE) constitute accepted markers for early diagnosis of prostate cancer, but they fail to accurately determine the precise location and extent of the lesion(s). Biopsy on the other hand, offers better localization of the suspected lesion through histological analysis but is an invasive technique and approximately $10 \%$ of small foci of cancer are not detected, since there is a high probability that the biopsy needle might miss the lesion. In recent years, MRI examination has been suggested for improved visualization and localization of prostate central gland (CG), peripheral zone (PZ) and seminal vesicles (SV) Fig 1 . With the majority of cancerous lesions appearing in the peripheral zone (70\%) [2], MRI provides valuable pathologic and anatomical information which is owed to the fact that, unlike ultrasound, it has a well-known ability in representing soft water-based tissues such as the prostate gland and seminal vesicles [3]. However, although this technique is very sensitive

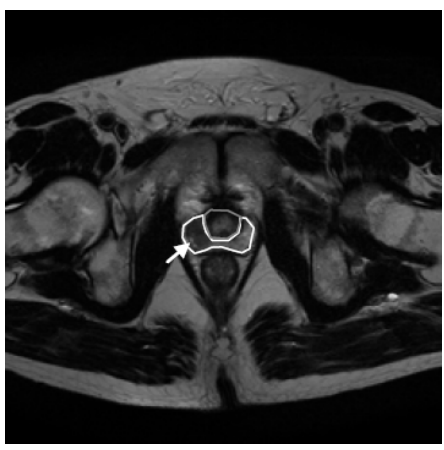

(a)

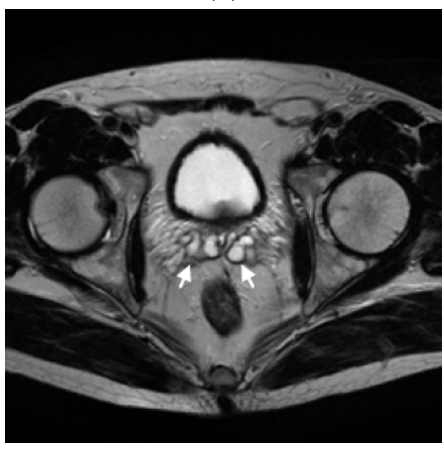

(b)

Fig. 1. (a) Axial T2-weighted image showing the central (gray contour) and peripheral zone (white contour) of a 61 year old patient. The low intensity (dark) signal on the left (white arrow) indicates the existence of suspicious lesion - in this case prostate carcinoma. The remaining part of the peripheral zone returns a high intensity signal, a feature of normal tissue. (b) Axial T2-weighted image showing the seminal vesicles of the same patient (white arrows). The returned high intensity signal indicates healthy tissue.

in detecting focal abnormalities in the prostate, its specificity in distinguishing between malignant and benign tissue and other abnormal structures is still unsatisfactory with a reported specificity of $43 \%$ [4]. Its ability to detect prostate carcinomas is also limited due to the fact that nodular hyperplasia and bleeding after biopsy examination can cause reduced signal intensity in the peripheral zone, a characteristic that is often mistaken for a suspicious lesion. 

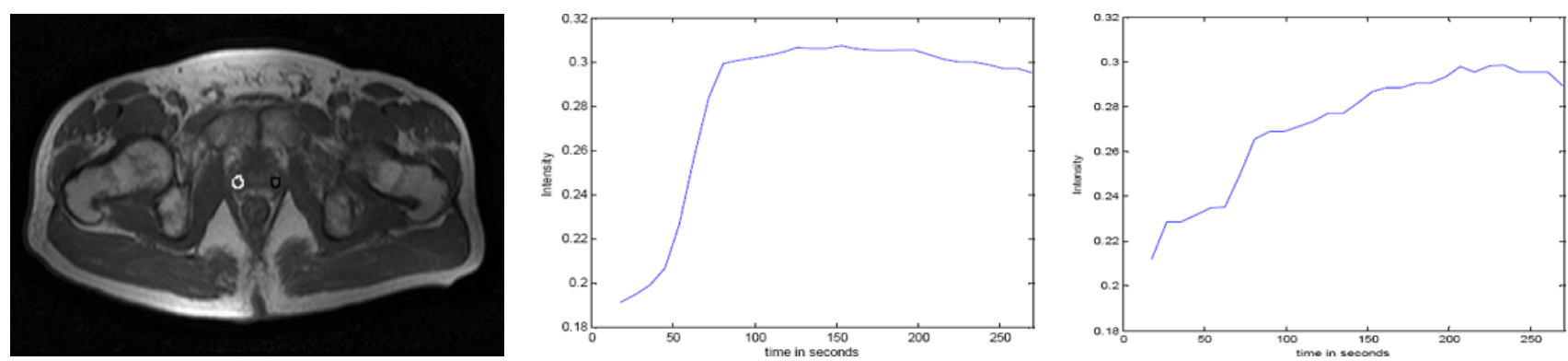

Fig. 2. (a) Axial DCE image, first out of a series of 30 DCE images. The white contour on the left indicates a malignat area whereas the black contour on the right indicates a benign tissue area. (b) Curve showing the mean dynamic response of the pixels within the white contour malignant area. It can be seen that the curve exhibits early rapid enhancement and then becomes almost steady to a maximum value, indicating high vascularity of the affected area which allows higher permeability of the contrast agent in the blood. This pattern is very common to cancerous lesions (c) Curve showing the mean dynamic response of the pixels within the black contour area. In this case the enhancement progresses slowly and the curve continues to rise almost until the end of the monitored time interval. This pattern is indicative of the less vascular normal or benign tissue of the prostate.

Recent advances in MRI techniques have made possible the acquisition of temporal information associated with the angiogenesis of hypervascular tumors through the use of Dynamic Contrast Enhanced MRI. The difference in enhancement patterns between tumor and benign tissue after intravenous injection of a contrast agent (e.g. gadolinium), has shown the potential of DCE MRIs to map the distribution of cancer within the prostate [5], [6]-[9]. Fig 2 demonstrates typical enhancement patterns for benign and malignant tissue within the peripheral zone of the prostate. Such enhancement patterns are also known as dynamic curves. Although magnetic resonance imaging has been in clinical use for more than a decade [10][14], only few works on computer aided detection of prostate cancer have been found [15], [16] that utilize MRIs. All of them concentrate on the analysis of morphological features which are extracted from T2-weighted images and associated T2 mapping methods.

In this work, extending our previous results in [19], we examine a classification procedure that utilizes features from both morphological and DCE images to create the corresponding clusters for benign and malignant tissue. Two are the main distinctions between this work and our previous work in [19]:

1) We apply a feature generation transform [20] that reduces the dimension of the feature vectors. Such a procedure, offers the potential to remove information redundancies which usually exist in the original set of features. Furthermore, we have noticed that this dimensionality reduction, significantly improves the probability that the EM algorithm (employed for training) converges to a satisfactory solution. Also, we noticed that this dimensionality reduction leads the classification system to obtain better generalization abilities, a result that is in accordance to [20].

2) More simulation results are provided. In particular, Receiver Operating Characteristic (ROC) curves are also given for the examined systems in conjunction to the Correct Classification Rates (CCRs). Furthermore, a computer search method is used here to aid us in the selection of the numbers of clusters per category that should be used.

Numerical results provided here strengthen the claim made in [19] that fusion of characteristics form DCE and T2 weighted MRIs offer the potential to improve the accuracy of the automated detection system, as compared to using features only from one imaging modality.

\section{Methology}

\section{A. System Overview}

The architecture of the computer-aided system for prostate cancer detection is shown in Fig 3. An MRI scanner is used for data acquisition. The data consists of axial T2-weighted morphological and axial DCE images that correspond to the same MR slice. Since the 30 DCE images are acquired over a period of 270 seconds, a motion correction algorithm is applied to correct possible micro-motion of the patients organs [19]. Furthermore, an algorithm for aligning the DCE images to the corresponding T2-weighted ones was used, so as to make possible the fusion of features between the two imaging modalities [19]. Depending on whether the system operates in training mode or in diagnostic mode, an expert radiologist or a possibly less experienced user employs the respective graphical user interface, shown with dashed borders in Fig 3. In training mode, the experienced doctor marks benign and/or malignant areas on the T2 MRI images. These images constitute the ground truth images. In diagnostic mode, a user selects a Region of Interest (ROI) for which automatically generated advice is desired. In either case, feature vectors, using information from both imaging modalities, are extracted for the desired area. 


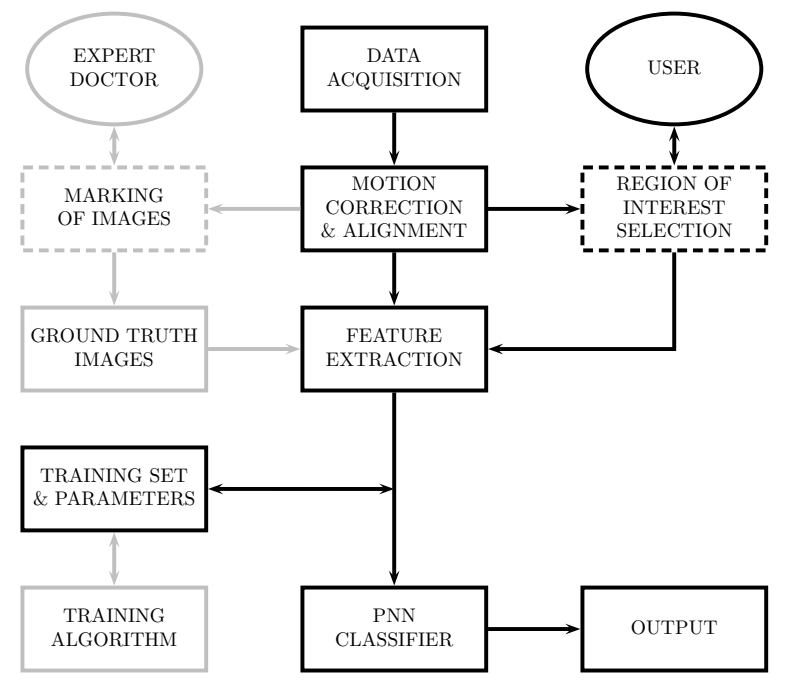

Fig. 3. Overview of the examined Computer Aided Detection System. The sub-modules that appear as light grey are only used during the training phase.

In the sequel, the extracted feature vectors are either fed to the previously trained classification algorithm (diagnostic mode) or are incorporated into the training set (training mode). The parameters of the classification system computed during the training phase are stored to a database along with the feature vectors that constitute the training set. The output of the system is a decision metric for each one of the feature vectors at the input of the Probabilistic Neural Network. In the following paragraphs, we analyze the sub-modules of the computer-aided detection system in greater detail.

\section{B. Data Acquisition}

The image acquisition was performed on a 1.5 Tesla MRI scanner using a body coil, on patients with biopsy proven, clinically localized prostate cancer. All chosen patients had no prior radiotherapy or other treatment which would alter T2-weighted intensities and/or dynamic behavior. The prostate gland of each patient was segmented in eight axial MRI slices, each having a $6 \mathrm{~mm}$ thickness. The distance between subsequent slices was $1.2 \mathrm{~mm}$. The types of the acquired images were T2-weighted morphological images and DCE images. All patients have been injected with a contrast agent prior to the scanning of the DCE examination.

The acquired dynamic series consist of thirty images for each of the eight slices of the prostate gland, with a temporal gap of 9 seconds between them, resulting to a total of 240 images. The size of the DCE images is $256 \times 256$ pixels per image and the pixel spatial resolution is $1.5 \mathrm{~mm} \times 1.5 \mathrm{~mm}$. The corresponding T2-weighted morphological images were also generated for each of the eight slices, with a spatial resolution of $0.5 \mathrm{~mm}$ per pixel and size $512 \times 512$ pixels per image. The lack of equal resolution on morphological and DCE images is owed to the fact that the DCE images should provide a sufficient temporal resolution that is traded-off against spatial resolution. Moreover, the field of view during the acquisition of the DCE images is larger so as to include areas around the prostate that would be prone to metastases.

\section{Motion Correction and Alignment}

MR images are known to be corrupted by Rician distributed noise caused by the thermal agitation of electrons/ions of the receiver coil and attached electronics as well the electrolytes in the patients body [23]. Wavelet domain thresholding techniques, have been employed for the elimination of such noise which offer the increased capability of keeping important image characteristics, such as edges, almost unaltered [22]. However, the difficulty in such methods lies in the accurate estimation of the unknown noise variance. Here, we tackled this problem by employing the Minimum Absolute Deviation (MAD) method [22].

The 30 DCE images are then processed through a motion correction algorithm [19] to reduce the effects of patient / organs motion that may have occurred during image acquisition.

Finally, the alignment procedure between the T1-weighted and T2-weighted images will have to be performed. However, since different RF excitation pulses are used to produce the DCE and T2-weighted morphological images to emphasize on different tissue properties [21], it can be deduced that their intensity values cannot directly be used for alignment purposes. Therefore, features independent of the contrast weighting must be employed [19]. Such features were extracted by exploiting the fact that MRIs of the prostate are almost symmetrical with respect to a vertical axis [19].

\section{Generation of Ground Truth Images}

The ground truth images for tumor and benign tissue were generated with the aid of biopsy results and radiological assessment. Two expert radiologists manually labeled suspected tumor areas on the peripheral zone on the axial T2-weighted morphological images. These labels have been compared with biopsy results on the peripheral zone to indicate the correct position of suspected tumors. Only regions marked positive in both biopsy reports and radiologists labeling procedure were considered as valid tumor ground truth labels. Similar areas have been labeled by experts to indicate the position of benign tissue. The labeled areas were used in the training procedure of the proposed system.

\section{E. Feature Extraction}

The aligned DCE and T2 weighted images are used for the production of a set of feature vectors. A separate feature vector is generated for each $\mathrm{T} 2$ weighted voxel of the desired patient 
region. As already mentioned, such a region may either be an expert-marked region used for training or a user selected ROI for which automated advice is desired. Three types of feature vectors were analyzed during our study, namely (a) using features only from DCE images (b) using features only from T2-weighted images and (c) using fused features from both imaging modalities.

For each voxel of the DCE image, a dynamic curve can be extracted by using information from the 30 temporally separated DCE images that correspond to the same slice. Thus, one could select a $30 \times 1$ feature vector holding these intensity values as the feature vector. Although such an approach has been used in [19], it has two major drawbacks: (a) The resulting classification system will have a great number of parameters, and thus, will obtain weak generalization abilities [20]. (b) Training of the classification system will be difficult to converge to a good solution due to the large number of free parameters to be estimated. Thus, in this work, we propose to use the Discrete Cosine Transform (DCT) to perform dimensionality reduction of the $30 \times 1$ feature vectors. In particular, the DCT of each dynamic curve is computed and only the 4 low-frequency coefficients of the transform are used to construct a $4 \times 1$ feature vector.

For each voxel of the T2 weighted image, similarly to [19], we construct a $3 \times 1$ feature vector holding the intensity of the respective pixel, the variance of the intensities in a $3 \times 3$ neighborhood around the current pixel and the kurtosis of the intensities in the same region.

Finally, feature vectors using fused information from both imaging modalities involve the 4 DCT coefficients of each dynamic curve and the respective intensity value from the $\mathrm{T} 2$ weighted image. Thus, the dimensions of each fused feature vector is $5 \times 1$.

\section{F. Classification}

The proposed computer-aided detection system has utilized Probabilistic Neural Networks for the classification procedure. PNNs categorize a feature vector $\mathbf{x}$ into two categories $H_{a}$ (Benign) or $H_{b}$ (Malignant) implementing the general Bayesian rule:

$$
d(\mathbf{x})=\left\{\begin{array}{lll}
H_{a} & \text { if } & p_{a} c_{b} f_{a}(\mathbf{x})>p_{b} c_{a} f_{b}(\mathbf{x}) \\
H_{b} & \text { if } & p_{a} c_{b} f_{a}(\mathbf{x})<p_{b} c_{a} f_{b}(\mathbf{x})
\end{array}\right.
$$

where $p_{a}$ and $p_{b}$ denote the a-priori probabilities of the categories $H_{a}$ and $H_{b}$ respectively and $c_{a}\left(c_{b}\right)$ denotes the cost associated with the decision in favor for $H_{a}\left(H_{b}\right)$ while the actual decision should be in favor of $H_{b}\left(H_{a}\right)$. Also, $f_{a}(\mathbf{x})$ and $f_{b}(\mathbf{x})$ denote the probability density functions of the respective categories. The parameters $p_{a}, p_{b}, c_{a}, c_{b}$ are usually set by experts about the detection problem at hand.
Moreover, the unknown probability density functions of the categories should be estimated using training data. In [24], it is proposed to estimate a general pdf as a weighted sum of Gaussian densities. Thus, we may approximate the density $f_{a}(\mathbf{x})$ as

$$
f_{a}(\mathbf{x})=\frac{1}{M_{a}(2 \pi)^{N / 2}|\mathbf{C}|} \sum_{i=1}^{M_{a}} e^{-\frac{1}{2}\left(\mathbf{x}-\mathbf{x}_{a i}\right)^{T} \mathbf{C}^{-1}\left(\mathbf{x}-\mathbf{x}_{a i}\right)}
$$

where $\mathbf{x}_{a i}$ denote the mean vectors of each Gaussian (centroids), $\mathbf{C}$ is the $N \times N$ covariance matrix of each Gaussian kernel and $N$ is the dimension of the input vectors. $M_{a}$ is the number of kernels used, which constitutes also a parameter to be estimated. The density $f_{b}(\mathbf{x})$ and the parameter $M_{b}$ are defined accordingly. In this work, the centroids $\mathbf{x}_{a i}, \mathbf{x}_{b i}$ of the categories $H_{a}$ and $H_{b}$ respectively as well as the covariance matrix $\mathbf{C}$ were estimated using the Expectation Maximization algorithm according to [25].

\section{EXPERIMENTAL RESULTS}

The three examined classification systems (i.e. using fused features, only DCE features and only T2 features) have been investigated using 10 patient cases. Patients B1-B5 have benign tissue while patients M1-M5 have malignant areas. The recruited patients were subjected to both ultrasoundguided biopsy and MRI and they have been assessed by two independent expert radiologists. In order to assess the generalization ability of each of the systems, we organized our experiments in the following manner: Tests were performed by excluding a pair of patients from training (one with benign tissue and one with malignant tissue), which resulted in twenty five different combinations. For each such combination, the patients that were excluded from the training set were used for testing.

For the pattern recognition algorithm, the a-priori probabilities were set equal to $1 / 2$ and the respective costs were set equal to unity, i.e. no bias was induced to the classification system. The parameters $M_{a}$ and $M_{b}$ were selected by performing the training-testing procedure for values of $M_{a}$ and $M_{b}$ in the set $\{1,2,3,4\}$ and keeping those that yielded the best results (in terms of the average CCR).

Tables I-III show the average CCRs, over all 25 test cases, obtained by the three examined systems for various numbers of Gaussian kernels used to model the unknown pdf of the two categories. The maximum average percentages obtained, appear in Tables I-III in boldface. The number of kernels for which the best CCRs were obtained give us a clue for the selection of the parameters $M_{a}$ and $M_{b}$.

Furthermore, it can be observed that the average CCRs obtained from employing both morphological and dynamic features outperform the average CCRs when the PNNs are trained with features employing information from only one 


\begin{tabular}{|c||c|c|c|c|}
\hline & $M_{b}=1$ & $M_{b}=2$ & $M_{b}=3$ & $M_{b}=4$ \\
\hline \hline$M_{a}=1$ & $83.50 \%$ & $84.84 \%$ & $84.90 \%$ & $83.77 \%$ \\
\hline$M_{a}=2$ & $83.46 \%$ & $86.27 \%$ & $85.37 \%$ & $84.28 \%$ \\
\hline$M_{a}=3$ & $84.90 \%$ & $\mathbf{8 6 . 9 2} \%$ & $86.14 \%$ & $85.57 \%$ \\
\hline$M_{a}=4$ & $84.64 \%$ & $85.60 \%$ & $85.51 \%$ & $84.99 \%$ \\
\hline
\end{tabular}

TABLE I

AVERAGE CCRS USING FUSED FEATURES AND VARIOUS NUMBERS OF CENTERS PER CATEGORY

\begin{tabular}{|c||c|c|c|c|}
\hline & $M_{b}=1$ & $M_{b}=2$ & $M_{b}=3$ & $M_{b}=4$ \\
\hline \hline$M_{a}=1$ & $74.28 \%$ & $68.27 \%$ & $67.59 \%$ & $67.67 \%$ \\
\hline$M_{a}=2$ & $72.86 \%$ & $72.07 \%$ & $71.93 \%$ & $73.98 \%$ \\
\hline$M_{a}=3$ & $72.76 \%$ & $71.56 \%$ & $69.87 \%$ & $72.26 \%$ \\
\hline$M_{a}=4$ & $74.38 \%$ & $\mathbf{7 5 . 3 9} \%$ & $70.40 \%$ & $69.89 \%$ \\
\hline
\end{tabular}

TABLE II

AVERAGE CCRS USING DCE FEATURES AND VARIOUS NUMBERS OF CENTERS PER CATEGORY

imaging modality. Interestingly, this seems to be the case regardless of the number of Gaussian kernels employed to model the respective probability density functions.

Tables IV-VI show the analytical CCRs for all twenty five test cases that correspond to the parameters $M_{a}$ and $M_{b}$ that yielded the maximum average CCR. Finally, in Fig. 4 the Receiver Operating Characteristic curves [20] of the three examined systems are shown. It can be observed that the system utilizing the fused features outperforms the other two, exhibiting higher sensitivity and specificity. Furthermore, the areas under the three ROC curves shown in Table VII show that the fusion of features from different imaging modalities increases the separation ability of the automated detection scheme.

\begin{tabular}{|c||c|c|c|c|}
\hline & $M_{b}=1$ & $M_{b}=2$ & $M_{b}=3$ & $M_{b}=4$ \\
\hline \hline$M_{a}=1$ & $77.60 \%$ & $77.61 \%$ & $\mathbf{7 7 . 6 2} \%$ & $77.57 \%$ \\
$M_{a}=2$ & $72.81 \%$ & $73.69 \%$ & $74.86 \%$ & $76.78 \%$ \\
$M_{a}=3$ & $75.63 \%$ & $75.69 \%$ & $74.89 \%$ & $75.70 \%$ \\
$M_{a}=4$ & $75.65 \%$ & $75.72 \%$ & $76.11 \%$ & $76.18 \%$ \\
\hline
\end{tabular}

TABLE III

AVERAGE CCRS USING T2 FEATURES AND VARIOUS NUMBERS OF CENTERS PER CATEGORY

\begin{tabular}{|c||c|c|c|c|c|}
\hline & M1 & M2 & M3 & M4 & M5 \\
\hline \hline B1 & $72.62 \%$ & $88.18 \%$ & $90.65 \%$ & $92.34 \%$ & $86.49 \%$ \\
\hline B2 & $68.71 \%$ & $85.27 \%$ & $100.00 \%$ & $99.77 \%$ & $92.04 \%$ \\
\hline B3 & $82.87 \%$ & $92.19 \%$ & $98.27 \%$ & $98.21 \%$ & $93.34 \%$ \\
\hline B4 & $66.29 \%$ & $83.35 \%$ & $80.02 \%$ & $85.69 \%$ & $78.15 \%$ \\
\hline B5 & $80.38 \%$ & $84.36 \%$ & $96.57 \%$ & $92.18 \%$ & $85.13 \%$ \\
\hline
\end{tabular}

TABLE IV

CCRS OBTAINED BY EXCLUDING ONE BENIGN AND ONE MALIGNANT PATIENT FROM TRAINING AND USING THEM FOR TESTING. FUSED FEATURES, $M_{a}=3, M_{b}=2$, AVERAGE CCR IS $86.92 \%$

\begin{tabular}{|c||c|c|c|c|c|}
\hline & M1 & M2 & M3 & M4 & M5 \\
\hline \hline B1 & $31.11 \%$ & $75.48 \%$ & $71.06 \%$ & $75.69 \%$ & $61.74 \%$ \\
\hline B2 & $49.36 \%$ & $87.47 \%$ & $88.86 \%$ & $95.81 \%$ & $85.96 \%$ \\
\hline B3 & $51.78 \%$ & $81.16 \%$ & $74.06 \%$ & $80.08 \%$ & $76.85 \%$ \\
\hline B4 & $51.41 \%$ & $79.63 \%$ & $85.13 \%$ & $83.13 \%$ & $75.54 \%$ \\
\hline B5 & $64.64 \%$ & $85.60 \%$ & $92.03 \%$ & $94.31 \%$ & $86.88 \%$ \\
\hline
\end{tabular}

TABLE V

CCRS OBTAINED BY EXLUDING ONE BENIGN AND ONE MALIGNANT PATIENT FROM TRAINING AND USING THEM FOR TESTING. DCE FEATURES, $M_{a}=4, M_{b}=2$, AVERAGe CCR IS $75.39 \%$

\begin{tabular}{|c||c|c|c|c|c|}
\hline & M1 & M2 & M3 & M4 & M5 \\
\hline \hline B1 & $78.13 \%$ & $63.21 \%$ & $92.47 \%$ & $94.95 \%$ & $81.42 \%$ \\
\hline B2 & $80.49 \%$ & $62.38 \%$ & $96.58 \%$ & $98.22 \%$ & $83.65 \%$ \\
\hline B3 & $85.46 \%$ & $67.43 \%$ & $95.24 \%$ & $96.50 \%$ & $86.80 \%$ \\
\hline B4 & $50.06 \%$ & $57.54 \%$ & $54.62 \%$ & $61.61 \%$ & $52.89 \%$ \\
\hline B5 & $78.04 \%$ & $66.62 \%$ & $86.81 \%$ & $88.98 \%$ & $80.35 \%$ \\
\hline
\end{tabular}

TABLE VI

CCRS OBTAINED BY EXCLUDING ONE BENIGN AND ONE MALIGNANT PATIENT FROM TRAINING AND USING THEM FOR TESTING. T2 FEATURES, $M_{a}=1, M_{b}=3$, Average CCR IS $77.62 \%$

\begin{tabular}{|c|c|}
\hline System & Area Under ROC \\
\hline \hline Using Fused Features & 0.8977 \\
\hline Using T1 Features & 0.7903 \\
\hline Using T2 Features & 0.8635 \\
\hline
\end{tabular}

TABLE VII

COMPARISON OF THE AREAS UNDER THE ROC CURVES IN FIG. 4

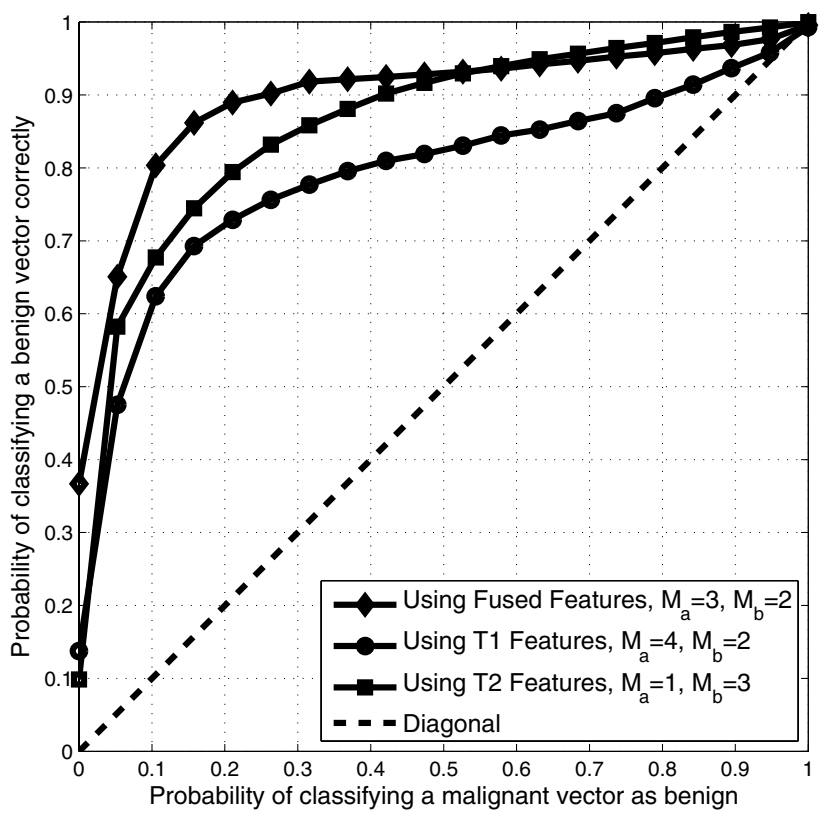

Fig. 4. Receiver Operating Characteristic curves of the three examined systems 


\section{CONCLUSiON}

This paper presented an overview of a computer-aided scheme for the detection of prostate cancer. The proposed system utilized information from morphological T2-weighted MRI images and DCE MRI images that were properly aligned so as to extract the features that composed the training vectors of the classification scheme. Probabilistic Neural Networks, trained using the Expectation Maximization algorithm, were employed for the detection of malignant or benign tissue of the prostate. Tests performed with 10 patient cases, showed that the use of fused features outperforms that of only morphologic or only dynamic features.

However, this work has shown only preliminary results. The potential of the proposed system has to be explored further with respect to different features that can be extracted from both of the aforementioned imaging modalities. Equally important, information from other MRI techniques, such as T2 ADC mapping methods [26] and magnetic resonance spectroscopy [27] might increase the separation ability between malignant and benign tissue even more. Moreover, the performance of other pattern recognition schemes such as the Support Vector Machines [28] has to be evaluated so as to determine a good combination of features and classifier for the problem at hand.

\section{ACKNOWLEDGMENT}

This research was supported by the Cyprus Research Promotion Foundation, by research contract PLHRO/0104/03. Special thanks to C. Economides, M.D. Radiologist, E. Eracleous, M. D. Radiologist, of Therissos MRI Center, Nicosia, Cyprus and C. Kouriefs, M. D. Urologist, Queen Elizabeth Hospital, London, UK for their continuous guidance on clinical issues in this work.

\section{REFERENCES}

[1] J. E. S Husband, R. H. Reznek, Imaging in Oncology, Oxford Press, 1998.

[2] C. L. Carrol, F. G. Sommer, J. E. McNeal, T. A. Stammey, "The abnormal prostate: MR Imaging at $1.5 \mathrm{~T}$ with histopathologic correlation", Radiologgy, 1987;163:521-525.

[3] Villeirs et.al, "Magnetic resonance imaging anatomy of the prostate and periprostatic area: a guide for radiotherapists", J. of the Eur. Soc. for Therapeutic Radiology,vol. 76, , pp. 99106, 2005.

[4] H. Carter, R. Brem, C. Tempany, A. Yang, J. Epstein, P. Walsh, and E. Zerhouni, "Nonpalpable prostate cancer: detection with MR imaging", Radiology 178, 5235251991.

[5] M.R. Engelbrecht et.al, "Discrimination of Prostate Cancer from normal peripheral zone and central gland tissue by using dynamic contrast enhanced MR imaging", J. of Radiology, vol. 229, , pp. 248254, 2003.

[6] A. R. Padhani, C. J Gapinsky, D. A. Macvicar, G J. Parker, J Sucking, P. B Revell, M. O. Leach, D. P. Dearnaley, J. E. Husband, "Dynamic Contrast Enhanced MRI of prostate cancer: correlation with morphology and tumor stage, histological grade and PSA", Clin. Radiol., 2000, 55:99109.
[7] L. W. Turnbull, D. L Buckley, L. S. Turnbull, G. P. Liney, A. J. Knowles, "Differentiation of prostatic canrcinoma and benign prostatic hyperplasia: correlation between dynamic Gd-DTPA-enhanced MR imaging and histopathology”, J. Magn Reson Imaging, 1999, 9:311-316.

[8] O. Rouviere, A. Raudrant, R. Ecohard, C. Colin-Pangaud, C. Pasquiou, R. Bouvier, J. M. Marechal, D. Lyonnet, "Characterization of timeenhancement curves of benign and malignant prostate tissue at dynamic MR imaging", Eur. Radiol, 2003, 13:931-942.

[9] P. Preziosi, A. Orlaccio, G. Di Gambattista, P. Di Renzi, L. Bortolotti, A. Fabiano, E. Cruciani, P. Pasqualetti, "Enhancement Patterns of Prostate Cancer in dynamic MRI”, Eur. Radiol, 2003, 13:925-930.

[10] M. D. Rifkin et al., "Comparison of magnetic resonance imaging and ultrasonography in staging early prostate cancer: Results of a multi-institutional cooperative trial", New Engl. J. Med., vol. 323, pp. 621626,1990

[11] S. Ikonen et al., "Magnetic resonance imaging of clinically localized prostatic cancer", J. Urol., vol. 159, no. 3, pp. 915919, 1998.

[12] S. Quinn, D. Franzini, T. Demlow, D. Rosencrantz, J. Kim, R. Hanna, and J. Szumowski, "MR imaging of prostate cancer with an endorectal surface coil technique: Correlation with whole-mount specimens", Radiol. ,vol. 190, pp. 323-327, 1994.

[13] M. L. Schiebler et al.,"Current role of MR imaging in staging of adenocarcinoma of the prostate", Radiol. , vol. 189, no. 2, pp. 339352, 1993.

[14] E. Outwater,M. Schiebler, J. Tomaszewski, M. Schnall, and H. Kressel, "Mucinous carcinomas involving the prostate: Atypical findings at MR imaging”, J. Magn. Reson. Imag., vol. 2, pp. 597600, 1992.

[15] A. Madabhushi, M. D. Feldman, D. N. Metaxas, J. Tomaszeweski, D. Chute "Automated Detection of Prostatic Adenocarcinoma from High Resolution Ex-Vivo MRI", IEEE Transactions on Medical Imaging, vol. 24, no. 12, December 2005, pp. 1611-1625.

[16] I. Chan, W.Wells, R. Mulkern, S. Harker, J. Zhang, K. Zou, S. Maier, C. Tempany, "Detection of prostate cancer by integration of line-scan diffusion, T2-mapping and T2-weighted magnetic resonance imaging; a multichannel statistical classifier", Med. Phys., pp. 23902398, 2003.

[17] D. F. Specht, "Probabilistic Neural Networks, Neural Networks", Neural Networks, Vol. 3, 1990, pp. 109-118.

[18] A. P. Dempster, N. M. Laird, D. B. Rubin, "Maximum Likelihood from incomplete data via the EM algorithm", Journal of the Royal Statistical Society (B), 39, 1-38, 1977.

[19] D. Ampeliotis, A. Antonakoudi, K. Berberidis, E. Z. Psarakis, "Detection Of Prostate Cancer Using Fused Information from T1 and T2 weighted Magnetic Resonance Images", IEEE Int. Conf. on Signal Processing and Communications, Nov. 24-27, Dubai - UAE, 2007

[20] S. Theodoridis, K. Koutroumbas, Pattern recognition ,2nd Edition, Academic Press, 2003.

[21] J. L. Prince and J. M. Links, Medical Imaging Signals and Systems, Pearson Prentice Hall Bioengineering, 2006.

[22] S. Mallat, A Wavelet Tour of Signal Processing, Academic Press, 1998.

[23] R. D. Nowak, "Wavelet Based Rician Noise Removal for Magnetic Resonance Imaging", IEEE Trans. On Image Processing, vol. 8, No. 8, pp. 14081419, October 1999.

[24] E. Parzen, "On estimation of probability density function and mode", Annals of Mathematics, 33, pp. 1065-1076, 1962.

[25] R. L. Streit, T. E. Luginbuhl, "Maximum Likelihood Training of Probabilistic Neural Networks", Neural Networks, 3, pp 109-118, 1994.

[26] D.-M. Koh, D. J. Collins, "Diffusion-Weighted MRI in the Body: Applications and Challenges in Oncology", American Journal of Roentgenology, 188, June2007, pp. 1622-1635.

[27] D. Pucar, A. Shukla-Dave, H. Hricak, C. S. Moskowitz, K. Kuroiwa, S. Olgac, L. E. Ebora, P. T. Scardino, J. A. Koutcher, K. L. Zakian, "Prostate Cancer: Correlation of MR Imaging and MR Spectroscopy with Pathologic Findings after Radiation TherapyInitial Experience", Radiology 2005;236:545-553

[28] C. J. C. Burges, "A Tutorial on Support Vector Machines for Pattern Recognition", Data Mining and Knowledge Discovery, 2, pp. 121-167, 1998, Kluwer Academic Publishers. 